\title{
Superfluid transition in the attractive Hofstadter-Hubbard model
}

\author{
R. O. Umucalılar and M. Iskin \\ Department of Physics, Koç University, Rumelifeneri Yolu, 34450 Sarlyer, Istanbul, Turkey
}

(Received 24 May 2016; published 9 August 2016)

\begin{abstract}
We consider a Fermi gas that is loaded onto a square optical lattice and subjected to a perpendicular artificial magnetic field, and determine its superfluid transition boundary by adopting a BCS-like mean-field approach in momentum space. The multiband structure of the single-particle Hofstadter spectrum is taken explicitly into account while deriving a generalized pairing equation. We present the numerical solutions as functions of the artificial magnetic flux, interaction strength, Zeeman field, chemical potential, and temperature, with a special emphasis on the roles played by the density of single-particle states and center-of-mass momentum of Cooper pairs.
\end{abstract}

DOI: 10.1103/PhysRevA.94.023611

\section{INTRODUCTION}

Experiments with ultracold atomic systems have incessantly progressed in the past two decades or so, since the creation of the very first Bose-Einstein condensate (BEC) with a dilute gas of bosonic atoms. Equipped with unique opportunities to control a wide range of parameters, these systems have successfully been employed in not only testing numerous theoretical models developed in the condensedmatter literature, but also studying new phenomena which do not have a direct analog in other fields [1]. For instance, by changing the effective interaction strength between atoms through what is known as magnetic Feshbach resonances, the so-called BCS-BEC crossover has been experimentally realized with a superfluid (SF) Fermi gas, although such a phenomenon was originally explored from a theoretical perspective in the context of high- $T_{c}$ superconductors [2]. Similarly, the basic mechanism for unconventional pairings that has been experimentally realized with a populationimbalanced SF Fermi gas was originally proposed as a mechanism for inhomogeneous superconductivity that is caused by the Zeeman-induced mismatch of the Fermi surfaces, long before the advent of atomic systems [3,4]. More recently, there has been fervent activity in the cold-atom community to realize quantum-Hall-like effects with charge-neutral atoms through the use of artificial gauge fields and synthetic dimensions [5].

In this paper, we are interested in a merger of these topics, i.e., we study the SF transition of a Fermi gas that is loaded onto a square optical lattice and subjected to a perpendicular artificial magnetic field in the context of the so-called attractive Hofstadter-Hubbard model. Limited aspects of this problem were investigated both in momentum space within the BCS-like mean-field approach, while paying attention to single-particle degeneracies [6,7], and in real space within the Bogoliubov-de Gennes formalism including the possibility of imbalanced chemical and/or vector potentials [8]. Here, we focus on determining the SF transition boundary as a function of the artificial magnetic flux, interaction strength, Zeeman field, chemical potential, and temperature. In comparison to the existing literature, we not only develop a better understanding of the pairing mechanism in momentum space, but also locate the transition boundary more precisely within the adopted approximations. We also examine the roles played by the density of single-particle states and center-of- mass (CoM) momentum of Cooper pairs on the transition boundary, providing clear insights into the intriguing reentrant superfluidity behavior found in the numerical solutions. We trace the origin of this reentrant behavior back to the strongly modified density of single-particle states in the presence of a magnetic flux. The magnetic flux splits the original band of the field-free case into several subbands [9]. As a result of such a change in the band structure, the density of single-particle states becomes a nonmonotonic function of energy, imposing a similar nonmonotonic behavior on the phase boundaries.

The rest of the paper is organized as follows. In Sec. II, we first introduce the attractive Hofstadter-Hubbard model, and then obtain a self-consistent equation for the SF transition boundary by tackling the model Hamiltonian with a BCS-like mean-field approach in momentum space. Our numerical results are given in Sec. III, where we present the phase boundaries in interaction strength-Zeeman field, interaction strength-chemical potential, and temperature-chemical potential planes for a number of magnetic flux values. We conclude the paper with a brief summary in Sec. IV. In addition, detailed derivations of the Hofstadter spectrum and generalized pairing equation are outlined, respectively, in Appendices A and B, and additional phase diagrams are included in Appendix C.

\section{MEAN-FIELD THEORY}

Our fundamental assumption is that the motion of a single particle in a tight-binding square optical lattice that is subjected to a perpendicular magnetic field is well described by the famous Hofstadter model,

$$
H_{B}=-t \sum_{\langle i j\rangle \sigma}\left(e^{i 2 \pi \phi_{i j}} c_{i \sigma}^{\dagger} c_{j \sigma}+\text { H.c. }\right),
$$

where $c_{i \sigma}^{\dagger}\left(c_{i \sigma}\right)$ creates (annihilates) a fermion with pseudospin $\sigma \equiv\{\uparrow, \downarrow\}$ at site $i$, H.c. is the Hermitian conjugate, $t>0$ is the hopping amplitude between nearest-neighbor sites $\langle i j\rangle$, and $\phi_{i j}=\left(1 / \phi_{0}\right) \int_{\mathbf{r}_{j}}^{\mathbf{r}_{i}} \mathbf{A} \cdot d \mathbf{r}$ is the spin-independent phase factor the particle acquires while hopping from site $j$ to $i$. Here, $\phi_{0}=\hbar / q_{0}$ is the effective magnetic-flux quantum with $q_{0}$ the effective charge, and $\mathbf{A}=(0, B x)$ is the vector potential in the Landau gauge with $B$ the magnitude of the effective magnetic field. Note that neither $q_{0}$ nor $B$ corresponds to a physical quantity by itself in atomic systems that are engineered to 
simulate artificial gauge fields; only their product is physically meaningful. When the particle traverses a loop encircling a unit cell of the lattice, its wave function acquires the AharonovBohm phase factor $e^{i 2 \pi \alpha}$, where $\alpha=B a^{2} / \phi_{0}$ is the flux quanta per unit cell with $a \rightarrow 1$ the lattice constant. As we outline in Appendix A for completeness, when $\alpha$ is a rational fraction $p / q$ with $p$ and $q$ co-prime integers, the tight-binding $s$ band of the single-particle spectrum in the field-free case splits into $q$ subbands, yielding the so-called Hofstadter butterfly which is a self-similar function of $\alpha$ [9]. The noninteracting Hamiltonian $H_{0}=H_{B}-\sum_{i \sigma} \mu_{\sigma} n_{i \sigma}$ in the grand-canonical ensemble can equivalently be expressed as $H_{0}=H_{B}-\mu \sum_{i}\left(n_{i \uparrow}+n_{i \downarrow}\right)-$ $h \sum_{i}\left(n_{i \uparrow}-n_{i \downarrow}\right)$, where $n_{i \sigma}=c_{i \sigma}^{\dagger} c_{i \sigma}$ is the number operator, $\mu=\left(\mu_{\uparrow}+\mu_{\downarrow}\right) / 2$ is the average chemical potential, and $h=$ $\left(\mu_{\uparrow}-\mu_{\downarrow}\right) / 2$ can be interpreted as an out-of-plane Zeeman field.

We restrict ourselves to on-site atom-atom interactions that are described by the attractive Hubbard Hamiltonian $H_{I}=U \sum_{i} c_{i \uparrow}^{\dagger} c_{i \downarrow}^{\dagger} c_{i \downarrow} c_{i \uparrow}$, where $U \leqslant 0$. Adopting a BCS-like mean-field approximation for pairing, i.e., assuming that the fluctuations of the quadratic operators $c_{i \downarrow} c_{i \uparrow}$ are small in comparison to their equilibrium values, we may decouple $H_{I}$ as

$$
H_{I} \approx-\sum_{i}\left(\Delta_{i} c_{i \uparrow}^{\dagger} c_{i \downarrow}^{\dagger}+\Delta_{i}^{*} c_{i \downarrow} c_{i \uparrow}+\frac{\left|\Delta_{i}\right|^{2}}{U}\right),
$$

where the complex order parameter $\Delta_{i}=U\left\langle c_{i \uparrow} c_{i \downarrow}\right\rangle$ describes the on-site atom-atom correlations in thermal equilibrium as denoted by the thermal average $\langle\ldots\rangle$. The SF phase is characterized by $\Delta_{i} \neq 0$ at least for some $i$. When $\Delta_{i}=0$ for all $i$, the spin- $\sigma$ particles are either a normal Fermi gas or form a band insulator, depending on their thermal average numbers determined by $N_{i \sigma}=\left\langle n_{i \sigma}\right\rangle$. Due to the Pauli exclusion principle, $N_{\sigma}=\sum_{i} N_{i \sigma}$ can at most be the total number of lattice sites $M=M_{x} M_{y}$, corresponding to a fully occupied spectrum for any given $\alpha$. However, when the number of fully occupied magnetic subbands for spin- $\sigma$ particles is precisely an integer $s \leqslant q$ such that $N_{\sigma} / M=s / q$, or, equivalently, $\mu_{\sigma}$ is inside the corresponding single-particle energy gap, the particles form a band insulator. Otherwise, they are normal.

A compact closed-form expression for the SF transition boundary can be obtained in momentum $(\mathbf{k})$ space where it is relatively easier to diagonalize $H_{B}$. For this purpose, we introduce the k-space operators,

$$
c_{\mathbf{k} \beta \sigma}=\sqrt{\frac{q}{M_{x} M_{y}}} \sum_{s=0}^{M_{x} / q} \sum_{i_{y}=0}^{M_{y}} c_{s \beta i_{y} \sigma} e^{-i k_{x} s q} e^{-i k_{y} i_{y}},
$$

where $M_{x}$ and $M_{y}$ are, respectively, the number of lattice sites along the $x$ and $y$ directions with periodic boundary conditions in mind, and $\mathbf{k}=\left(k_{x}, k_{y}\right)$ is the momentum vector. Here, the real-space coordinate of site $i$ is expressed as $\mathbf{r}_{i}=$ $\left(i_{x}, i_{y}\right)$, where $i_{x}=s q+\beta$ with $s=0, \ldots, M_{x} / q$ denoting the location of the enlarged $(q \times 1)$ unit cell in the lattice and $\beta=0, \ldots, q-1$ denoting a particular site inside the enlarged unit cell. Since such choice of an enlarged unit cell restores the translational symmetry of the original lattice for the particular Landau gauge of interest, it allows us to retain the
Bloch description of the eigenstates with a reduced (magnetic) Brillouin zone (MBZ): $k_{x} \in[-\pi / q, \pi / q)$ and $k_{y} \in[-\pi, \pi)$ $[9,10]$.

Using Eq. (3) in Eq. (1), we obtain $H_{B}=$ $\sum_{\mathbf{k} \sigma} \sum_{\alpha \beta} c_{\mathbf{k} \alpha \sigma}^{\dagger} H_{\mathbf{k} \sigma}^{\alpha \beta} c_{\mathbf{k} \beta \sigma}$, where the matrix elements $H_{\mathbf{k} \sigma}^{\alpha \beta}$ are explicitly given in Appendix A. Diagonalization of this $q \times q$ matrix yields $q$ eigenvalues $\varepsilon_{\mathbf{k} n \sigma}$ for a given $\mathbf{k}$, with $n=$ $1, \ldots, q$ corresponding to $q$ subbands that split from the original field-free band. Note that the single-particle spectrum $\varepsilon_{\mathbf{k} n \sigma}=\varepsilon_{\mathbf{k} n}$ is spin independent. Using the band operators $d_{\mathbf{k} n \sigma}$ defined through the relation $c_{\mathbf{k} \beta \sigma}=\sum_{n} g_{\beta}^{n}(\mathbf{k}) d_{\mathbf{k} n \sigma}$, where $g_{\beta}^{n}(\mathbf{k})$ is the $\beta$ th component of the $n$th eigenvector of the single-particle problem with energy $\varepsilon_{\mathbf{k} n}$, and including $\mu_{\sigma}$, the noninteracting Hamiltonian finally reads as

$$
H_{0}=\sum_{\mathbf{k} n \sigma} \epsilon_{\mathbf{k} n \sigma} d_{\mathbf{k} n \sigma}^{\dagger} d_{\mathbf{k} n \sigma}
$$

where $\epsilon_{\mathbf{k} n \sigma}=\varepsilon_{\mathbf{k} n}-\mu_{\sigma}$ with $\mathbf{k}$ restricted to the first MBZ. Following a similar procedure, the Hamiltonian (2) can be written in $\mathbf{k}$ space as

$$
\begin{aligned}
H_{I}= & -\sum_{l \beta}\left\{\sum _ { n n ^ { \prime } \mathbf { k } } \left[\Delta_{\beta}^{l} g_{\beta}^{n *}\left(\mathbf{k}_{+}^{l}\right) g_{\beta}^{n^{\prime} *}\left(\mathbf{k}_{-}^{l}\right)\right.\right. \\
& \left.\left.\times d_{\mathbf{k}_{+}^{l} n \uparrow}^{\dagger} d_{\mathbf{k}_{-}^{l} n^{\prime} \downarrow}^{\dagger}+\text { H.c. }\right]+\frac{M}{q U}\left|\Delta_{\beta}^{l}\right|^{2}\right\},
\end{aligned}
$$

where the complex coefficients $\Delta_{\beta}^{l}=-(q U / M)$ $\sum_{n n^{\prime} \mathbf{k}} g_{\beta}^{n}\left(\mathbf{k}_{+}^{l}\right) g_{\beta}^{n^{\prime}}\left(\mathbf{k}_{-}^{l}\right)\left\langle d_{\mathbf{k}_{-}^{l} n^{\prime} \downarrow} d_{\mathbf{k}_{+}^{l} n \uparrow}\right\rangle$ are defined in such a way that $\Delta_{i}=\sum_{l} \Delta_{\beta}^{l} e^{i\left(Q_{l x} s+Q_{l y} i_{y}\right)}$. Here, $\mathbf{k}_{ \pm}^{l}= \pm \mathbf{k}+\mathbf{Q}_{l} / 2$ with $\mathbf{Q}_{l}=\left(Q_{l x}, Q_{l y}\right)$ the CoM momentum of Cooper pairs. While all possible CoM momenta must, in principle, be allowed in the calculations, such a task is not numerically tractable for arbitrary $\alpha$. For this reason, we limit our numerical calculations mainly to BCS-like pairings and consider a finite set $\mathbf{Q}_{l}=(0,2 \pi l p / q)$ with $l=0, \ldots, q-1$. Finite CoM pairing $\mathbf{Q}_{l}=(0,2 \pi l p / q)$ with $l \neq 0$, in addition to the usual BCS pairing with $\mathbf{Q}_{l}=(0,0)$, needs to be taken into account due to the degeneracy of the single-particle energies in any given band $n$ for momenta $\mathbf{k}$ and $\mathbf{k}+\mathbf{Q}_{l}$, i.e., $\varepsilon_{\mathbf{k} n}=\varepsilon_{\mathbf{k}+\mathbf{Q}_{l}, n}$ [6]. In the absence of a Zeeman field, we do not expect this limitation to a finite set of CoM momenta to have any effect on the SF transition boundary of interest here, even though the SF order parameter may slightly be affected by it deeper into the SF region. In the presence of imbalanced populations, while we expect this limitation to have some but minor influence on the SF transition boundary, we note that extending the calculation to Fulde-Ferrell-Larkin-Ovchinnikov (FFLO)-like pairings (e.g., by including additional CoM momenta to explicitly take the Zeeman-induced mismatch of the Fermi surfaces into account) may lead to a dramatic improvement in case a more accurate real-space description of the SF order parameter is desired.

Under these approximations and noting that all of the coefficients $\Delta_{\beta}^{l}$ are expected to be small in the vicinity of the SF transition boundary, $H_{I}$ may be treated as a perturbative correction to $H_{0}$. Using the first-order perturbation theory outlined in Appendix B, we obtain a compact expression for 
the generalized pairing equation,

$$
\begin{aligned}
\Delta_{\beta}^{l}= & -\frac{q U}{M} \sum_{n n^{\prime} \mathbf{k} \beta^{\prime}} g_{\beta}^{n}\left(\mathbf{k}_{+}^{l}\right) g_{\beta}^{n^{\prime}}\left(\mathbf{k}_{-}^{l}\right) g_{\beta^{\prime}}^{n *}\left(\mathbf{k}_{+}^{l}\right) g_{\beta^{\prime}}^{n^{\prime} *}\left(\mathbf{k}_{-}^{l}\right) \\
& \times \Delta_{\beta^{\prime}}^{l} \frac{1-f\left(\epsilon_{\mathbf{k}_{+}^{l} n \uparrow}\right)-f\left(\epsilon_{\mathbf{k}_{-}^{l} n^{\prime} \downarrow}\right)}{\epsilon_{\mathbf{k}_{+}^{l} n \uparrow}+\epsilon_{\mathbf{k}_{-}^{l} n^{\prime} \downarrow}},
\end{aligned}
$$

which determines the SF transition boundary for a given $\mathbf{Q}_{l}$. Here, $f(x)=1 /\left[e^{x /\left(k_{B} T\right)}+1\right]$ is the usual Fermi-Dirac distribution function, with $k_{B}$ the Boltzmann constant and $T$ the temperature. Note that Eq. (6) has to be supplied simultaneously with the number equations $N_{\sigma}=\sum_{n \mathbf{k}} f\left(\epsilon_{n \mathbf{k} \sigma}\right)$, forming a complete set of self-consistency equations for $\Delta^{l}=\left(\Delta_{0}^{l}, \ldots, \Delta_{q-1}^{l}\right)$ and $\mu_{\sigma}$. It is convenient to express Eq. (6) in the form of a matrix-eigenvalue equation, where $\Delta_{\beta}^{l}=\sum_{\beta^{\prime}} \mathbb{M}_{\beta \beta^{\prime}}^{l} \Delta_{\beta^{\prime}}^{l}$ or, equivalently, $\Delta^{l}=\mathbb{M}^{l} \Delta^{l}$, from which the condition for a nontrivial yet arbitrarily small $\Delta^{l}$ solution is determined by $\operatorname{setting} \operatorname{det}\left(\mathbb{I}-\mathbb{M}^{l}\right)=0$, with $\mathbb{I}$ the identity matrix. In the case of multiple solutions for $U_{c}^{l}$ and $T_{c}^{l}$ that are allowed by the determinant condition, we ultimately identify $U_{c}=\max \left\{U_{c}^{l}\right\}$ (or, equivalently, $\left|U_{c}\right|=\min \left\{\left|U_{c}^{l}\right|\right\}$ ) as the critical interaction strength and $T_{c}=\max \left\{T_{c}^{l}\right\}$ as the critical temperature of the system.

These critical parameters depend sensitively on $\alpha$ directly through the resultant density of single-particle states $D(\varepsilon)=$ $d \mathcal{N}(\varepsilon) / d \varepsilon$, where $\mathcal{N}(\varepsilon)$ is the number of states per unit area with energy smaller than $\varepsilon$, and it can be calculated by simply counting the number of states $\Delta \mathcal{N}(\varepsilon)$ contained in a small interval of energy $[\varepsilon, \varepsilon+\Delta \varepsilon]$ with fixed $\Delta \varepsilon$. Since the spectrum is symmetric around $\varepsilon=0$, we only consider $\varepsilon \geqslant 0$, as discussed below.

\section{NUMERICAL RESULTS}

First of all, in the absence of a magnetic field $(B \rightarrow 0$ or $\alpha \rightarrow 0$ ), which can equivalently be accounted for by taking $p=q=1$ and $l=\beta=0$, the determinant condition reduces to the usual expression $-M / U_{c}=\sum_{\mathbf{k}}\left[1-f\left(\epsilon_{\mathbf{k} \uparrow}\right)-\right.$ $f\left(\epsilon_{-\mathbf{k} \downarrow}\right] /\left(\epsilon_{\mathbf{k} \uparrow}+\epsilon_{-\mathbf{k} \downarrow}\right)$, where $\epsilon_{ \pm \mathbf{k} \sigma}=-2 t\left(\cos k_{x}+\cos k_{y}\right)-$ $\mu_{\sigma}$ is the usual tight-binding spectrum shifted by the chemical potential. We recall that FFLO-like pairings [4] are not considered in this work for the simplicity of the followup discussion. In Fig. 1(a), we show that $\left|U_{c}\right|$ is a monotonously increasing function of the Zeeman field $h$, which closely follows the monotonous decrease of $D(\varepsilon)$ with increasing $\varepsilon$ $(\geqslant 0)$ that is presented in Fig. 1(b). This is simply because, since $h \neq 0$ changes the effective chemical potentials for spin- $\uparrow$ and $-\downarrow$ particles as $\mu_{\uparrow, \downarrow}=\mu \pm h$, it directly affects the available number of states near the Fermi surface involved in pairing. When $D(\varepsilon)$ gets lower (higher), the formation of Cooper pairs is facilitated with a relatively large (small) $U_{c}$, which is a generic observation valid also in the presence of a magnetic field.

As the first example of a case with nonvanishing magnetic flux, we consider $\alpha=1 / 2$ and set $\mu=0$ for simplicity. The original field-free band splits into two bands that are touching each other at $\varepsilon=0$. The singular peak of $D(\varepsilon)$ that is seen in Fig. 2(b) at $\varepsilon=2 t$ is due to a van Hove singularity, and it is directly reflected as a dip in $\left|U_{c}\right|$ precisely at $h=2 t$ that is
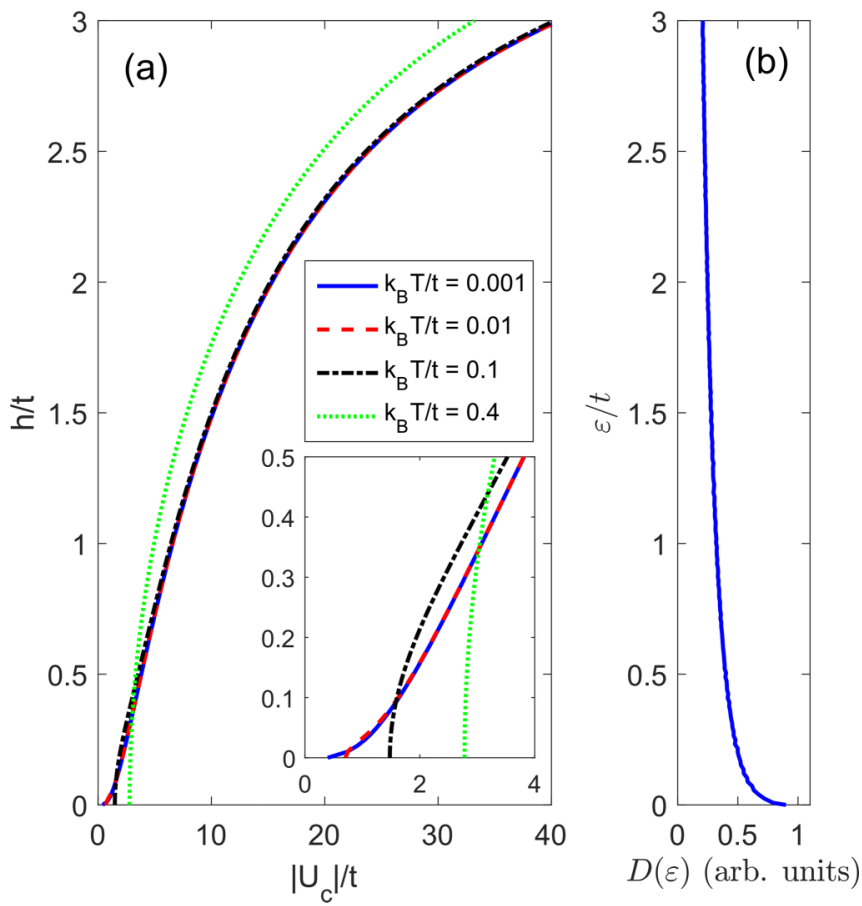

FIG. 1. (a) Critical interaction strength $\left|U_{c}\right| / t$ vs the Zeeman field $h / t$ with $\alpha=1 / 1$ and $\mu=0$ for various temperatures $T$. The inset is a close-up for $h \leqslant 0.5 t$. $\left|U_{c}\right| / t$ at $h=0$ increases with $T$. (b) Density of states $D(\varepsilon)$ in arbitrary units.
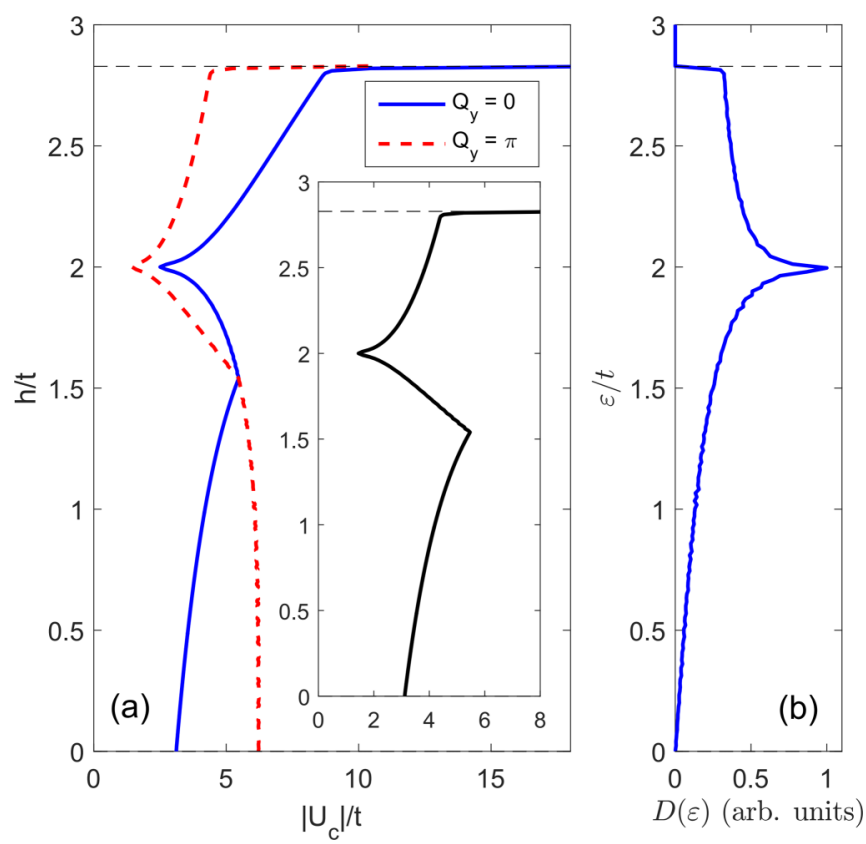

FIG. 2. (a) Critical interaction strength $\left|U_{c}\right| / t$ vs the Zeeman field $h / t$ with $\alpha=1 / 2, \mu=0$, and $k_{B} T=0.005 t$. The solid blue curve is for $\mathbf{Q}=(0,0)$ and the red dashed curve is for $\mathbf{Q}=(0, \pi)$. The curve in the inset traces the minimum value of the two curves at each $h / t$. (b) Density of states $D(\varepsilon)$ in arbitrary units. Horizontal dashed lines show the band edges including $\varepsilon=0$. 

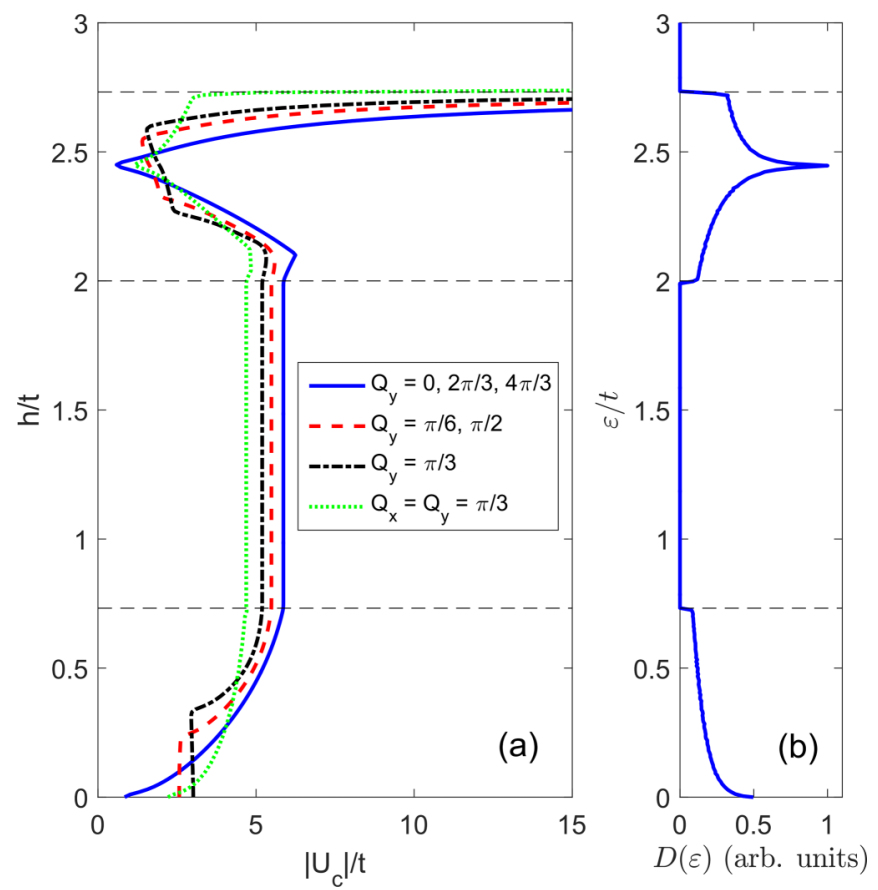

FIG. 3. (a) Critical interaction strength $\left|U_{c}\right| / t$ vs the Zeeman field $h / t$ with $\alpha=1 / 3, \mu=0$, and $k_{B} T=0.005 t$. The solid blue curve is the phase boundary obtained for $\mathbf{Q}_{l}=(0,2 \pi l / 3)$, with $l=0,1,2$. The dashed red curve is for $\mathbf{Q}=(0, \pi / 6)$ and $\mathbf{Q}=(0, \pi / 2)$, the dashdotted black curve is for $\mathbf{Q}=(0, \pi / 3)$, and the dotted green curve is for $\mathbf{Q}=(\pi / 3, \pi / 3)$. (b) Density of states $D(\varepsilon)$ in arbitrary units. Horizontal dashed lines show the band edges.

shown in Fig. 2(a). More importantly, the figure inset illustrates that $U_{c}$ is determined either by $\mathbf{Q}_{0}=(0,0)$ or $\mathbf{Q}_{1}=(0, \pi)$, depending on the particular value of $h$.

As a second example, shown in Fig. 3, we consider $\alpha=$ $1 / 3$ and again set $\mu=0$. While there are three subbands in the spectrum, only the highest band and half of the middle band are seen in Fig. 3(b) since $D(\varepsilon)$ is restricted to $\varepsilon \geqslant 0$. Figure 3(a) shows that $U_{c}^{l}$ are degenerate functions of $h$ for $\mathbf{Q}_{l}=(0,2 \pi l / 3)$, with $l=0,1$, and 2 , and the calculated dips correspond again to the peaks of $D(\varepsilon)$. It would be curious to check whether the set $\mathbf{Q}_{l}$ yields degenerate solutions for any $\alpha$ with odd denominators, e.g., see Appendix C for $\alpha=1 / 5$. When $\mu_{\uparrow}=-\mu_{\downarrow}=h$ is inside the single-particle band gap, i.e., between $0.73 t$ and $2 t,\left|U_{c}\right|$ remains constant until new pairing possibilities appear as $\mu_{\sigma}$ crosses over to the upper or lower bands, leading to the observed reentrant superfluidity behavior. Note in the gapped region that the ground state of the system is a band insulator with fillings $N_{\uparrow} / M=2 / 3$ and $N_{\downarrow} / M=1 / 3$ for $|U|<\left|U_{c}\right|$.

In Fig. 3(a), we also present the transition boundary for three additional values of CoM momenta, namely $\mathbf{Q}=(0, \pi / 6)$, $\mathbf{Q}=(0, \pi / 3)$, and $\mathbf{Q}=(0, \pi / 2)$. While $\left|U_{c}\right|$ is smaller for our original set $\mathbf{Q}_{l}=(0,2 \pi l / 3)$, with $l=0,1$ and 2 near the peaks of $D(\varepsilon)$, these additional CoM momenta lead, in general, to close but lower $\left|U_{c}\right|$. Although we do not systematically study the dependence of $U_{c}$ on the additional CoM momentum for a given $\alpha$ and $h$, here we provide the plausibility argument for this observation.
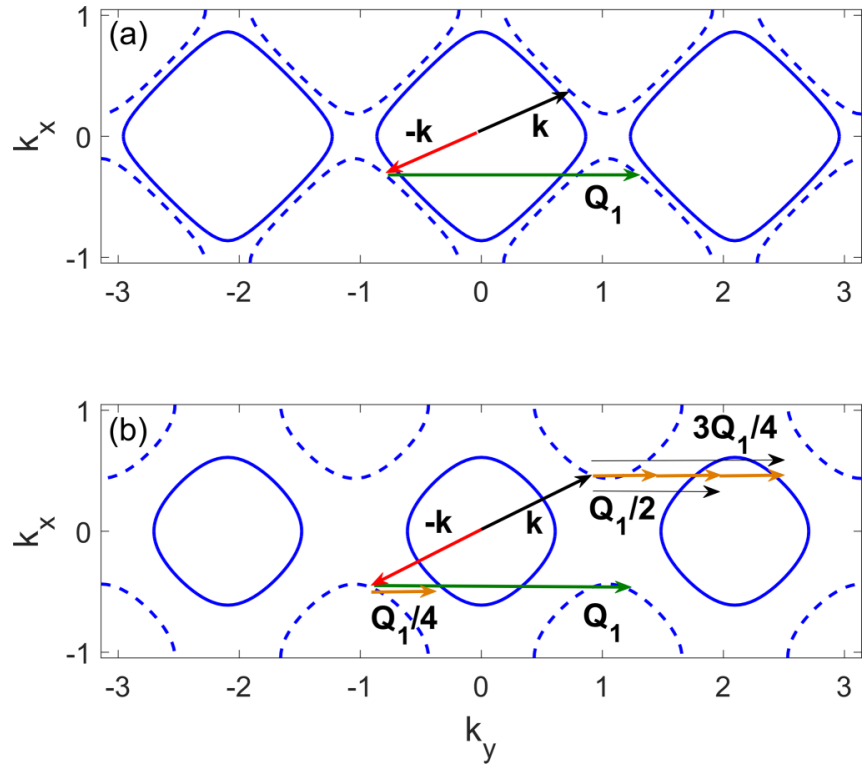

FIG. 4. (a) The most plausible pairing is sketched between a spin- $\uparrow$ particle with momentum $-\mathbf{k}+\mathbf{Q}_{1}$ and a spin- $\downarrow$ particle with momentum $\mathbf{k}$ for $\alpha=1 / 3$ and $\mu=0$. Fermi surfaces $\mu_{\uparrow}=0.05 t$ and $\mu_{\downarrow}=-0.05 t$ are shown by solid and dashed lines, respectively. (b) A similar sketch for $h=0.25 t$ shows that the CoM momenta $\mathbf{Q}_{1} / 4, \mathbf{Q}_{1} / 2$, and $3 \mathbf{Q}_{1} / 4$ give better matching of the Fermi surfaces in comparison to the original $\mathbf{Q}_{1}$.

In Fig. 4, we set $\alpha=1 / 3$ and $\mu=0$, and sketch the Fermi surfaces of spin- $\uparrow$ (solid curves) and spin- $\downarrow$ (dashed curves) particles for two $h$ values, showing a number of pairing possibilities inside the middle band of the spectrum. For small $h=0.05 t$, Fig. 4(a) shows that a spin- $\downarrow$ particle with momentum $\mathbf{k}$ can be easily coupled to a spin- $\uparrow$ particle with momentum $-\mathbf{k}+\mathbf{Q}_{1}$. Note that even though it is possible to find an arbitrary $\mathbf{Q}$ for a given $\mathbf{k}$ with the property of carrying $-\mathbf{k}$ close to a solid curve, $\mathbf{Q}_{1}$ and its integer multiples have this property for all $\mathbf{k}$. Therefore, we expect such pairings to be enhanced over other types of pairing. For a larger $h=0.25 t$, Fig. 4(b) clearly shows that the pairing of a spin- $\downarrow$ particle with momentum $\mathbf{k}$ and a spin- $\uparrow$ particle with momentum $-\mathbf{k}+\mathbf{Q}_{1}$ is energetically much harder than the previous case, instead of which pairings with $\mathbf{Q}_{1} / 4, \mathbf{Q}_{1} / 2$ and $3 \mathbf{Q}_{1} / 4$ are relatively easier with a better match of the Fermi surfaces. Furthermore, relaxing the condition on the vanishing $x$ component of the CoM momenta, e.g., $\mathbf{Q}_{D}=(\pi / 3, \pi / 3)$, allows for a nesting vector with perfect overlap between the Fermi surfaces, despite an energy gap. Hence, we expect such CoM momentum vectors to be optimal when they are relevant. For example, Fig. 3(a) shows that $\mathbf{Q}_{D}$ not only leads to the lowest $\left|U_{c}\right|$ when $h$ is inside a band gap, but also its result may deviate significantly from other possibilities when $\mu_{\uparrow, \downarrow}$ is close to the highest or lowest band edge. A comprehensive analysis of such generalized pairing schemes is beyond the scope of this work, and we defer it to a future one.

The third example is shown in Fig. 5, where we consider $\alpha=1 / 4$ and set $\mu=0$. The original field-free band splits into a total of four bands, two of which are touching each other at $\varepsilon=0$. Figure 5(a) shows that $U_{c}^{l}$ solutions corresponding to 

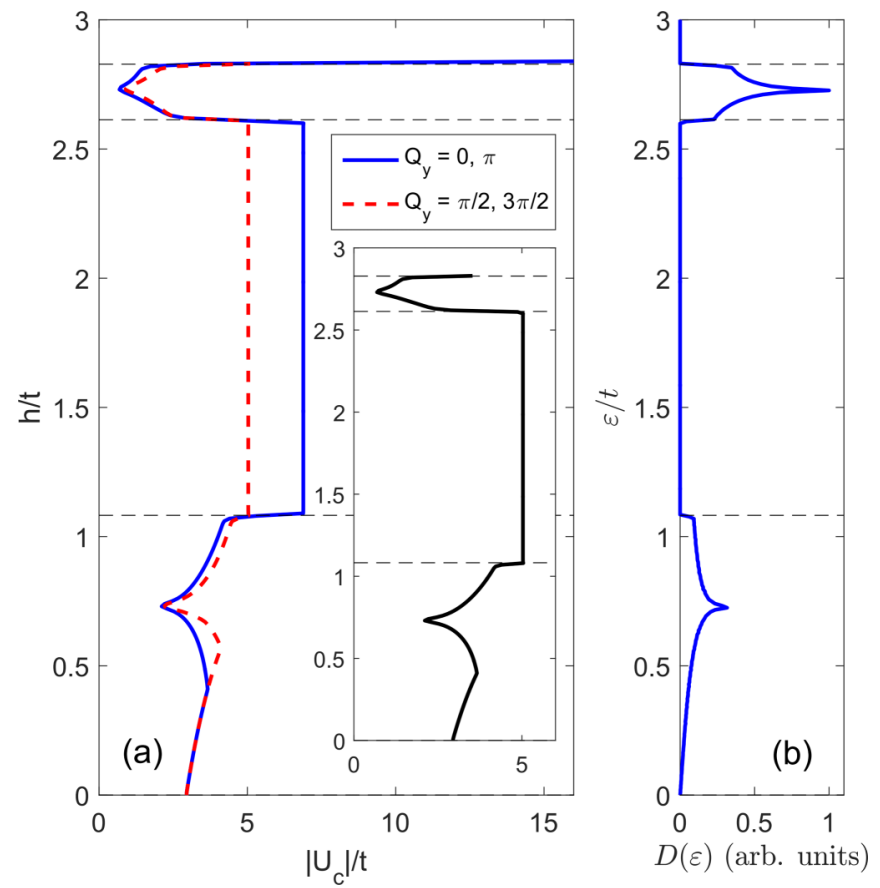

FIG. 5. (a) Critical interaction strength $\left|U_{c}\right| / t$ vs the Zeeman field $h / t$ with $\alpha=1 / 4, \mu=0$, and $k_{B} T=0.005 t$. The solid blue curve is for $\mathbf{Q}=(0,0)$ and $\mathbf{Q}=(0, \pi)$; the red dashed curve is for $\mathbf{Q}=(0, \pi / 2)$ and $\mathbf{Q}=(0,3 \pi / 2)$. The inset shows the minimum value of the two curves at each $h / t$. (b) Density of states $D(\varepsilon)$ in arbitrary units. Horizontal dashed lines show the band edges including $\varepsilon=0$.

the $l=(0,2)$ and $l=(1,3)$ CoM momentum $\mathbf{Q}_{l}=(0,2 \pi l / 4)$ are degenerate functions of $h$, and it would be curious to check whether such a grouping of solutions is possible for any $\alpha$ with even denominators, e.g., see Appendix $\mathrm{C}$ for $\alpha=1 / 6$. In accordance with the previous discussion, while $\left|U_{c}\right|$ again remains a constant when $\mu_{\sigma}= \pm h$ are inside the energy gap between the two highest single-particle bands, its ground state is a band insulator with fillings $N_{\uparrow} / M=3 / 4$ and $N_{\downarrow} / M=$ $1 / 4$ for $|U|<\left|U_{c}\right|$.

For completeness, next we again consider $\alpha=1 / 4$, but analyze the effects of $\mu \neq 0$ by setting $\mu=-t$. Since $\mu_{\sigma}$ is lowered by $-t$, Fig. 6 shows that $\left|U_{c}\right|$ has a single dip at around $h \simeq 1.7 t$ within the range of $h$ presented. This peak corresponds to the enhanced pairing between a spin- $\uparrow$ particle with $\mu_{\uparrow} \simeq 0.7 t$ from the middle band of the spectrum and a spin- $\downarrow$ particle with $\mu_{\downarrow} \simeq-2.7 t$ from the lowest band. Similar to the $\mu=0$ case, we again see that $U_{c}^{l}$ solutions corresponding to the $l=(0,2)$ and $l=(1,3) \mathrm{CoM}$ momentum $\mathbf{Q}_{l}=(0,2 \pi l / 4)$ are degenerate functions of $h$.

The pairing equation (6) can also be used to determine $\left|U_{c}\right|$ as a function of $\mu$. For instance, we consider a populationbalanced system with three distinct $\alpha=1 / 3,1 / 4$, and $1 / 5$ values in Fig. 7, where we set $h=0$ for simplicity. The results are symmetric around $\mu=0$ due to the particle-hole symmetry of the Hamiltonian. In all three cases, we observe that the local minima of $\left|U_{c}\right|$ coincide intuitively with the local maxima of $D(\varepsilon)$. This follows from the fact that if $\mu_{\uparrow}=\mu_{\downarrow}$ is inside a band and there exist a large number of states in the vicinity of $\mu$ available for pairing, then $\left|U_{c}\right|$ is small. However, as $D(\varepsilon)$

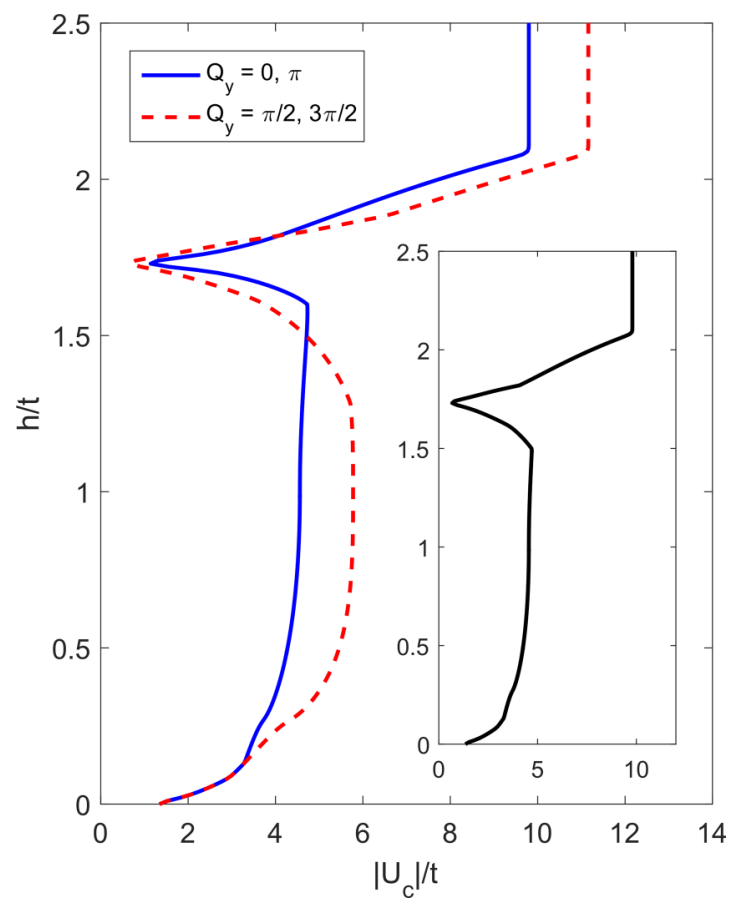

FIG. 6. (a) Critical interaction strength $\left|U_{c}\right| / t$ vs the Zeeman field $h / t$ with $\alpha=1 / 4, \mu=-t$, and $k_{B} T=0.005 t$. The solid blue curve is for $\mathbf{Q}=(0,0)$ and $\mathbf{Q}=(0, \pi)$; the red dashed curve is for $\mathbf{Q}=(0, \pi / 2)$ and $\mathbf{Q}=(0,3 \pi / 2)$. The inset shows the minimum value of the two curves at each $h / t$.

vanishes when $\mu$ enters a band gap, $\left|U_{c}\right|$ gets larger, attaining its maximum value roughly in the middle of the gap. It is remarkable that the maximum values of $\left|U_{c}\right|$ in the topmost band gaps of the spectra range somewhere between $4.3 t$ and $4.9 t$ without much variation.
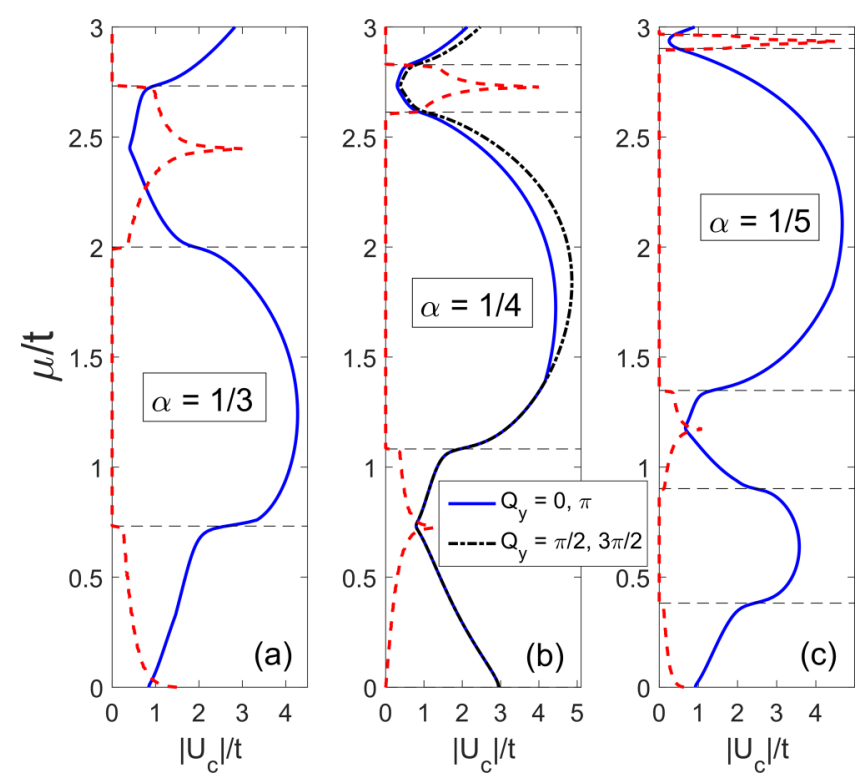

FIG. 7. Critical interaction strength $\left|U_{c}\right| / t$ vs the chemical potential $\mu / t$ for (a) $\alpha=1 / 3$, (b) $\alpha=1 / 4$, and (c) $\alpha=1 / 5$. Here, $h=0$ and $k_{B} T=0.005 t$. The density of states $D(\varepsilon)$ is also shown in arbitrary units by a red dashed curve. Horizontal dashed lines mark the band edges including $\varepsilon=0$ in (b). 


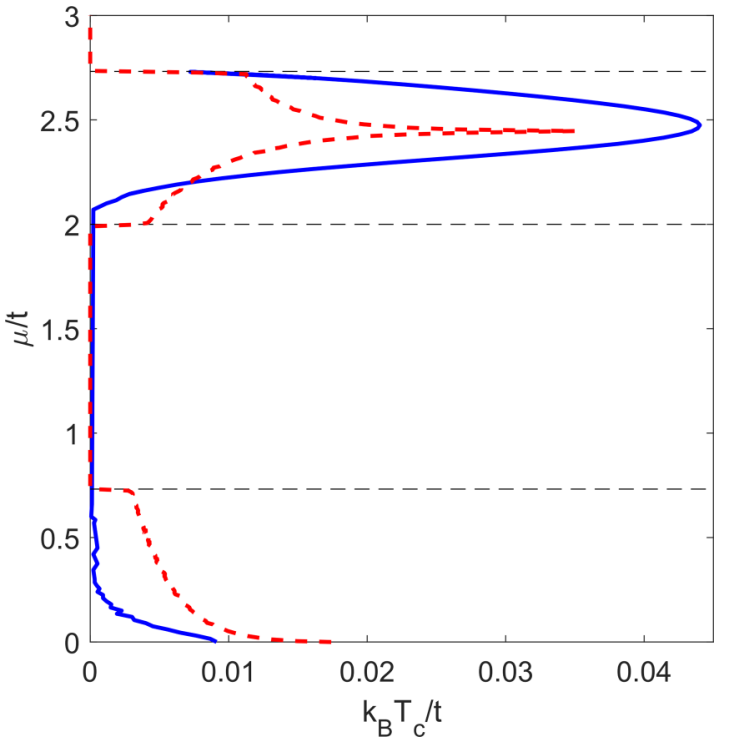

FIG. 8. Critical temperature $k_{B} T_{c} / t$ as a function of the chemical potential $\mu / t$. Here, $\alpha=1 / 3,|U|=t$, and $h=0$. The density of states $D(\varepsilon)$ is also shown in arbitrary units by a red dashed curve. Horizontal dashed lines mark the band edges.

As a last application of Eq. (6), we determine $T_{c}$ in Fig. 8 as a function of $\mu$, where we consider $\alpha=1 / 3$, and set $|U|=t$ and $h=0$. As increasing $T$ weakens the SF state by breaking the Cooper pairs, the enhanced pairing due to high $D(\varepsilon)$ is eventually beaten by higher $T$. When $\mu$ is inside the band gap, the system remains as an insulator even at $T \sim 0$ for the chosen small value of $|U|$. However, when $\mu$ is inside a band, the system remains as a SF up to a critical $T_{c}$, the peak values of which coincide with the peak values of $D(\varepsilon)$ around the middles of the bands.

\section{CONCLUSION}

To summarize, here we thoroughly analyzed the superfluid transition in the attractive Hofstadter-Hubbard model, as an attempt to describe neutral Fermi gases that are loaded onto square optical lattices and subjected to perpendicular and uniform artificial magnetic fields. Adopting a BCS-like meanfield approach in momentum space, we derived a generalized pairing equation in the vicinity of the superfluid transition. We solved this equation for the critical interaction strength and critical temperature as functions of the Zeeman field and chemical potential by taking primarily into account the finite center-of-mass momentum pairing caused by the degeneracies of the single-particle Hofstadter spectrum. The nonmonotonic variations of the critical interaction strength and critical temperature are traced back to the sharp changes in the density of single-particle states and to the multiple bands of the Hofstadter spectrum, justifying the reentrant superfluidity behavior found in the phase diagrams.

An extension of this study would be to more precisely determine the contribution of Cooper pairs with additional center-of-mass momentum in building up especially the superfluid state. This may be accomplished through an optimization procedure which fully accounts for the interplay between the Zeeman field and the complex band structure arising from the artificial magnetic field. As another avenue, our analysis for a square lattice can be extended to different lattice geometries such as triangular or honeycomb ones in light of the recent experiments which demonstrated the possibility of deforming different lattice types into one another by tuning lattice parameters [11]. In particular, it would be interesting to study how the topological transitions [12] that could be effected in such tunable lattices change the phase boundaries under the combined action of a complex band structure and population imbalance.

\section{ACKNOWLEDGMENTS}

R.O.U. is supported by the TÜBITAK BIDEB 2232 Program. M.I. acknowledges funding from TÜBITAK Grant No. 1001-114F232 and the BAGEP award of the Turkish Science Academy.

\section{APPENDIX A: SINGLE-PARTICLE SPECTRUM}

The single-particle spectrum is determined by diagonalizing the $q \times q$ matrix,

$$
\mathbb{H}_{\mathbf{k} \sigma}=\mathbb{H}_{\mathbf{k}}=\left(\begin{array}{cccccc}
D_{1} & F & 0 & . & 0 & C \\
F^{*} & D_{2} & F & 0 & \cdot & 0 \\
0 & \ddots & \ddots & \ddots & 0 & . \\
\cdot & 0 & F^{*} & D_{m} & F & 0 \\
0 & . & 0 & \ddots & \ddots & \ddots \\
C^{*} & 0 & . & 0 & F^{*} & D_{q}
\end{array}\right),
$$

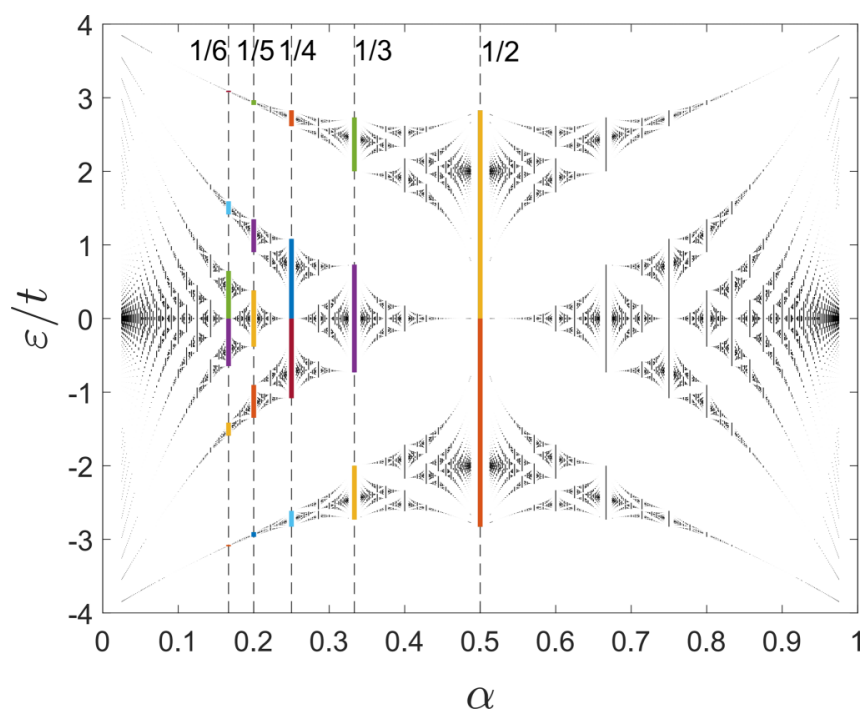

FIG. 9. Hofstadter butterfly spectrum displaying the singleparticle energy $\varepsilon / t$ as a function of the magnetic flux quanta per unit cell $\alpha=p / q$. For each $\alpha$, there are a total of $q$ energy bands. Vertical dashed lines correspond to $\alpha$ values considered in this work. Each band at such an $\alpha$ value is represented by a different color. When $q$ is even, two bands touch each other at $\varepsilon=0$. 
where $D_{m}=-2 t \cos \left(2 \pi m \alpha-k_{y}\right), \quad F=-t, \quad$ and $\quad C=$ $-t e^{-i q k_{x}}$. Note that reversing the sign of $\alpha \rightarrow-\alpha$ changes the diagonal elements to $D_{m}^{\prime}=-2 t \cos \left(2 \pi m \alpha+k_{y}\right)$, having no effect on our results since such a change corresponds to reversing the direction of the magnetic field. In addition, a basis transformation of the form $c_{\mathbf{k} \beta \sigma} \rightarrow c_{\mathbf{k} \beta \sigma} e^{ \pm i \beta k_{x}}$ changes $F$ to $F^{\prime}=-t e^{ \pm i k_{x}}$ and $C$ to $C^{\prime}=-t e^{\mp i k_{x}}$, again having no effect on our results. Representations of the Hofstadter matrix (A1) with the primed quantities are occasionally encountered in the literature [10]. In Fig. 9, we show the Hofstadter butterfly spectrum as determined by the eigenvalues of the matrix (A1) for all $\mathbf{k}$ in the first MBZ.

\section{APPENDIX B: GENERALIZED PAIRING EQUATION}

For completeness, here we follow Poole et al. [13] and briefly outline the derivation of Eq. (6). For an alternative method of derivation, see de Gennes [14]. We work in the interaction picture with $\tau=i t$ the imaginary time, and treat the interaction Hamiltonian (5) as a perturbation.

The propagator $\kappa(\tau)$ satisfies

$$
-\frac{d \kappa(\tau)}{d \tau}=H_{I}(\tau) \kappa(\tau),
$$

where $H_{I}(\tau)$ is the interaction Hamiltonian $H_{I}$ in the interaction picture,

$$
H_{I}(\tau)=e^{\tau H_{0}} H_{I} e^{-\tau H_{0}}
$$

By integrating Eq. (B1) to first order in $H_{I}$, we find

$$
\kappa(\tau) \simeq 1-\int_{0}^{\tau} H_{I}\left(\tau^{\prime}\right) d \tau^{\prime} .
$$
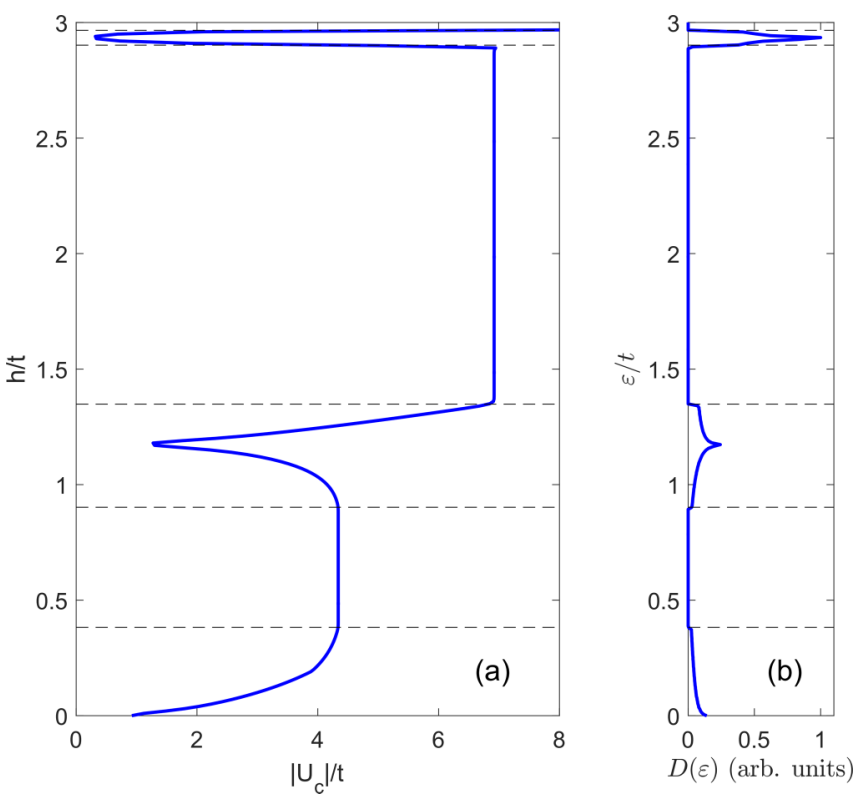

FIG. 10. (a) Critical interaction strength $\left|U_{c}\right| / t$ vs the Zeeman field $h / t$ with $\alpha=1 / 5, \mu=0$, and $k_{B} T=0.005 t$. The phase boundary is degenerate for all $\mathbf{Q}_{l}=(0,2 \pi l / 5)$ with $l=0,1, \ldots, 4$. (b) Density of states $D(\varepsilon)$ in arbitrary units. Horizontal dashed lines show the band edges.
The inverse of the propagator in this approximation,

$$
\kappa^{-1}(\tau) \simeq 1+\int_{0}^{\tau} H_{I}\left(\tau^{\prime}\right) d \tau^{\prime},
$$

obeys the relation $\kappa^{-1}(\tau) \kappa(\tau)=1$ up to first order in $\Delta_{\beta}^{l}$.

In order to construct a self-consistent equation for $\Delta_{\beta}^{l}$, we need to determine the average value $\left\langle d_{\mathbf{k}_{-}^{l} n^{\prime} \downarrow} d_{\mathbf{k}_{+}^{l} n \uparrow}\right\rangle$ in terms of $\Delta_{\beta}^{l}$. For this purpose, using $\langle\ldots\rangle=Z^{-1} \operatorname{Tr}\left[e^{-\tau H} \ldots\right]$ with $Z$ the partition function, and the cyclic property of the trace, we find

$$
\left\langle d_{\mathbf{k}_{-}^{l} n^{\prime} \downarrow} d_{\mathbf{k}_{+}^{l} n \uparrow}\right\rangle=\left\langle d_{\mathbf{k}_{+}^{l} n \uparrow}(\tau) d_{\mathbf{k}_{-}^{l} n^{\prime} \downarrow}\right\rangle,
$$

where we define

$$
\begin{aligned}
d_{\mathbf{k}_{+}^{l} n \uparrow}(\tau) & \equiv e^{\tau H} d_{\mathbf{k}_{+}^{l} n \uparrow} e^{-\tau H} \\
& =e^{-\tau \epsilon_{\mathbf{k}_{+}^{l}}^{l}{ }^{\prime}} \kappa^{-1}(\tau) d_{\mathbf{k}_{+}^{l} n \uparrow} \kappa(\tau) .
\end{aligned}
$$

An explicit form for $d_{\mathbf{k}_{+}^{l} n \uparrow}(\tau)$ can be found by using Eqs. (B2) (B4) and (B6), and keeping terms up to first order in $\Delta_{\beta}^{l}$, leading to

$$
\begin{aligned}
d_{\mathbf{k}_{+}^{l} n \uparrow}(\tau)= & e^{-\tau \epsilon_{\mathbf{k}_{+}^{l} n \uparrow}}\left[d_{\mathbf{k}_{+}^{l} n \uparrow}+\sum_{n^{\prime} l^{\prime} \beta^{\prime}} \Delta_{\beta^{\prime}}^{l^{\prime}} g_{\beta^{\prime}}^{n *}\left(\mathbf{k}_{+}^{l}\right) g_{\beta^{\prime}}^{n^{\prime} *}\left(\mathbf{k}_{-}^{l}+\mathbf{Q}_{l^{\prime}}\right)\right. \\
& \times \frac{e^{\tau\left(\epsilon_{\mathbf{k}_{+}^{l} n \uparrow}+\epsilon_{\left.-\mathbf{k}_{+}^{l}+\mathbf{Q}_{l^{\prime}, n^{\prime} \downarrow}\right)}\right.}-1}{\epsilon_{\mathbf{k}_{+}^{l} n \uparrow}+\epsilon_{-\mathbf{k}_{+}^{l}+\mathbf{Q}_{l^{\prime}}, n^{\prime} \downarrow}} d_{\left.-\mathbf{k}_{+}^{l}+\mathbf{Q}_{l^{\prime}, n^{\prime} \downarrow}^{\dagger}\right]}^{\text {(B7) }}
\end{aligned}
$$

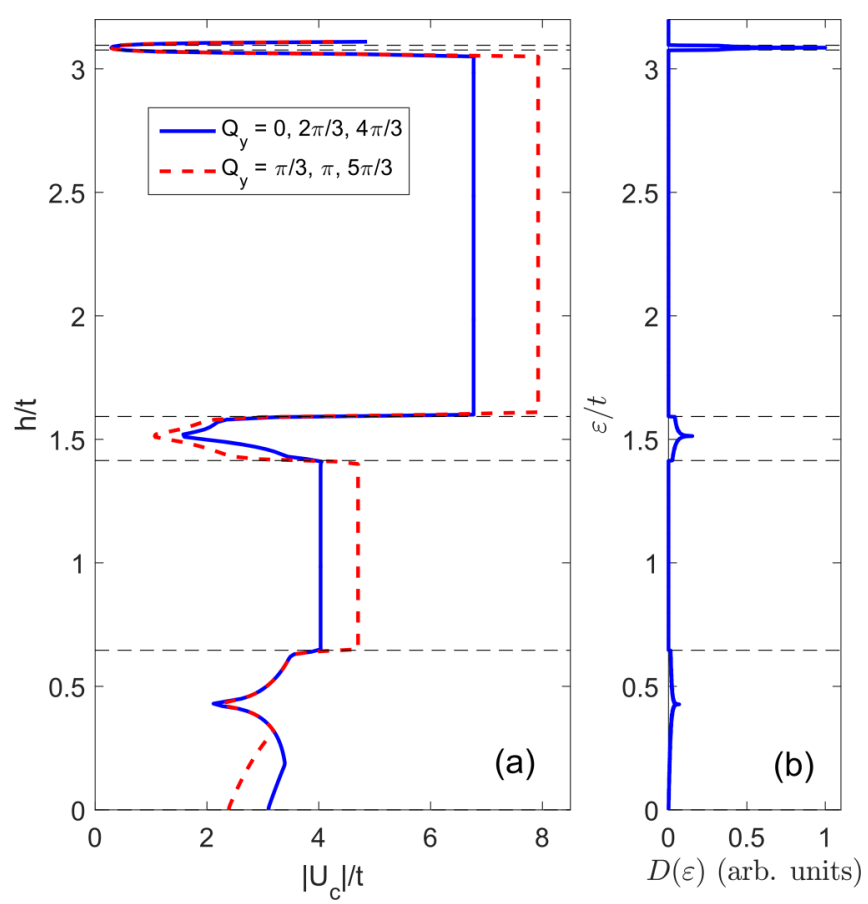

FIG. 11. (a) Critical interaction strength $\left|U_{c}\right| / t$ vs the Zeeman field $h / t$ with $\alpha=1 / 6, \mu=0$, and $k_{B} T=0.005 t$. The solid blue curve is for $\mathbf{Q}=(0,0), \mathbf{Q}=(0,2 \pi / 3)$, and $\mathbf{Q}=(0,4 \pi / 3)$; the red dashed curve is for $\mathbf{Q}=(0, \pi / 3), \mathbf{Q}=(0, \pi)$, and $\mathbf{Q}=(0,5 \pi / 3)$. (b) Density of states $D(\varepsilon)$ in arbitrary units. Horizontal dashed lines show the band edges including $\varepsilon=0$. 
Then, inserting Eq. (B7) into Eq. (B5), we find

$$
\begin{aligned}
\left\langle d_{\mathbf{k}_{-}^{l} n^{\prime} \downarrow} d_{\mathbf{k}_{+}^{l} n \uparrow}\right\rangle= & \sum_{\beta^{\prime}} \Delta_{\beta^{\prime}}^{l} g_{\beta^{\prime}}^{n *}\left(\mathbf{k}_{+}^{l}\right) g_{\beta^{\prime}}^{n^{\prime} *}\left(\mathbf{k}_{-}^{l}\right) f\left(\epsilon_{\mathbf{k}_{-}^{l} n^{\prime} \downarrow}\right) \\
& \times f\left(\epsilon_{\mathbf{k}_{+}^{l} n \uparrow}\right) \frac{e^{\tau\left(\epsilon_{\mathbf{k}_{+} n \uparrow \uparrow}+\epsilon_{\mathbf{k}_{-}^{l} n^{\prime} \downarrow}\right)}-1}{\epsilon_{\mathbf{k}_{+}^{l} n \uparrow}+\epsilon_{\mathbf{k}_{-}^{l} n^{\prime} \downarrow}},
\end{aligned}
$$

where $f(x)=1 /\left(e^{\tau x}+1\right)$. Here, we use $\left\langle d_{\alpha}^{\dagger} d_{\gamma}\right\rangle \simeq \delta_{\alpha \gamma} f\left(\epsilon_{\alpha}\right)$, which is valid up to first order. Finally, inserting Eq. (B8) into the definition of $\Delta_{\beta}^{l}$, rearranging the exponential terms, and replacing $\tau$ with $1 /\left(k_{B} T\right)$, we obtain the generalized pairing Eq. (6) given in the main text.

\section{APPENDIX C: PHASE DIAGRAMS FOR $\alpha=1 / 5$ AND $\alpha=1 / 6$}

For these lower magnetic flux values, since there are, respectively, five and six subbands in the energy spectrum,
Figs. 10 and 11 show much narrower bands in comparison to those presented in the main text with smaller $q$. In particular, the highest bands (as well as the lowest ones, which are not shown) of the $\alpha=1 / 5$ and $1 / 6$ spectra turn out to be very narrow, causing a sharp variation of $D(\varepsilon)$ with $\varepsilon$ and giving rise to a large dip in $\left|U_{c}\right|$ as a function of increasing $h$. A notable distinction between these two cases is that while $U_{c}^{l}$ are degenerate functions of $h$ for all $\mathbf{Q}_{l}=(0,2 \pi l p / q)$ with $l=0,1, \ldots, 4$ when $\alpha=1 / 5$, there are two distinct solution sets corresponding to $l=(0,2,4)$ and $l=(1,3,5)$ when $\alpha=1 / 6$.

As a final remark, we note that all of our numerical results for low $q=1,2, \ldots, 6$ values show that while $U_{c}^{l}$ are degenerate functions of $h$ for all $\mathbf{Q}_{l}$ when $q$ is odd, there are two distinct solution sets corresponding to $l=(0,2, \ldots, q-2)$ and $l=(1,3, \ldots, q-1)$ when $q$ is even. It would be curious to check whether this observation applies to arbitrary $q$ values in general.
[1] M. Lewenstein, A. Sanpera, V. Ahufinger, B. Damski, A. Sen De, and U. Sen, Ultracold atomic gases in optical lattices: mimicking condensed matter physics and beyond, Adv. Phys. 56, 243 (2007); I. Bloch, J. Dalibard, and W. Zwerger, Many-body physics with ultracold gases, Rev. Mod. Phys. 80, 885 (2008).

[2] S. Giorgini, L. P. Pitaevskii, and S. Stringari, Theory of ultracold atomic Fermi gases, Rev. Mod. Phys. 80, 1215 (2008); The BCS-BEC Crossover and the Unitary Fermi Gas, edited by W. Zwerger (Springer, New York, 2012).

[3] P. Fulde and R. A. Ferrell, Superconductivity in a strong spinexchange field, Phys. Rev. 135, A550 (1964); A. I. Larkin and Yu. N. Ovchinnikov, Nonuniform state of superconductors, Sov. Phys. JETP 20, 762 (1965).

[4] T. K. Koponen, T. Paananen, J.-P. Martikainen, M. R. Bakhtiari, and P. Törmä, FFLO state in 1-, 2- and 3-dimensional optical lattices combined with a non-uniform background potential, New J. Phys. 10, 045014 (2008).

[5] J. Dalibard, F. Gerbier, G. Juzeliūnas, and P. Öhberg, Colloquium: Artificial gauge potentials for neutral atoms, Rev. Mod. Phys. 83, 1523 (2011); N. Goldman, G. Juzeliūnas, P. Öhberg, and I. B. Spielman, Light-induced gauge fields for ultracold atoms, Rep. Prog. Phys. 77, 126401 (2014).

[6] H. Zhai, R. O. Umucalılar, and M. Ö. Oktel, Pairing and Vortex Lattices for Interacting Fermions in Optical Lattices with a Large Magnetic Field, Phys. Rev. Lett. 104, 145301 (2010). For a related work in the solid-state context, see Ref. [7].

[7] M. M. Maśka, Reentrant superconductivity in a strong applied field within the tight-binding model, Phys. Rev. B 66, 054533 (2002). This reference studies similar physics but with nearest- neighbor interactions, where only zero-CoM pairing is included without taking the eigenvectors of the single-particle spectrum into account in the numerical solutions.

[8] M. Iskin, Stripe-ordered superfluid and supersolid phases in the attractive Hofstadter-Hubbard model, Phys. Rev. A 91, 011601(R) (2015); Attractive Hofstadter-Hubbard model with imbalanced chemical and vector potentials, 91, 053606 (2015).

[9] D. R. Hofstadter, Energy levels and wave functions of Bloch electrons in rational and irrational magnetic fields, Phys. Rev. B 14, 2239 (1976).

[10] M. Kohmoto, Zero modes and the quantized Hall conductance of the two-dimensional lattice in a magnetic field, Phys. Rev. B 39, 11943 (1989).

[11] L. Tarruell, D. Greif, T. Uehlinger, G. Jotzu, and T. Esslinger, Creating, moving and merging Dirac points with a Fermi gas in a tunable honeycomb lattice, Nature (London) 483, 302 (2012); T. Uehlinger, G. Jotzu, M. Messer, D. Greif, W. Hofstetter, U. Bissbort, and T. Esslinger, Artificial Graphene with Tunable Interactions, Phys. Rev. Lett. 111, 185307 (2013).

[12] F. Yilmaz, F. N. Unal, and M. O. Oktel, Evolution of the Hofstadter butterfly in a tunable optical lattice, Phys. Rev. A 91, 063628 (2015); M. Iskin, Topological phase transitions on a triangular optical lattice with non-Abelian gauge fields, 93, 033632 (2016).

[13] C. P. Poole Jr., H. A. Farach, R. J. Creswick, and R. Prozorov, Superconductivity, 2nd ed. (Academic-Elsevier, New York, 2007).

[14] P. G. de Gennes, Superconductivity of Metals and Alloys (Westview Press, Boulder, CO, 1999). 